Aroueología Y Sociedad

№ $30,2015: 353-372$

ISSN: 0254-8062

RECIBIDO:JUNIO DE 2015

ACEPTADO: SETIEMBRE DE 2015

\title{
EXCAVACIONES EN LA PLATAFORMA Z1, PAMPA DE MOSQUITO. PRIMERA EVIDENCIA DEL ARCAICO TARDÍO EN EL VALLE MEDIO DEL RÍO JEQUETEPEQUE
}

\author{
EISEITSURUMI \\ UNIVERSIDAD DETOKIO \\ et@um.u-tokyo.ac.jp \\ CARLos A. Morales CASTRO \\ UnIVERSIDAD NACIONAL MAYOR DE SAN MARCOS \\ carlos_morales157@hotmail.com
}

\section{RESUMEN}

En las décadas de 1980, 1990 y la primera década de este siglo se realizaron intensas investigaciones arqueológicas en el valle medio del río Jequetepeque. Como resultado de estos trabajos se expuso una gran ocupación prehispánica, en especial, de asentamientos pertenecientes al periodo Formativo. Tanto así, que el pueblo de Tembladera fue conocido por su cerámica finamente elaborada (Alva 1986) durante el periodo Formativo y además a este periodo se le consideró como la ocupación más temprana conocida hasta el momento en este segmento del valle. Nuestras investigaciones realizadas en la Pampa de Mosquito, apoyados por elementos de arte rupestre y por datación radiocarbónica, dio como resultado que existió una ocupación más temprana vinculada al periodo Arcaico Tardío.

Palabras Claves: Jequetepeque, valle medio, Arcaico Tardío, plataformas, arte rupestre, datación radiocarbónica.

\section{AbSTRACT}

During 80 s and 90s, and the first decade of the present century, intense archaeological researches were made at the Jequetepeque middle valley. The results of these works showed prehispanic settlements, specially Formative settlements. Tembladera town was known by its finely crafted pottery (cf. Alva 1986) in the Formative period. This period was considered the oldest period in that part of the valley until now. Our researches in Pampa de Mosquito supported by rock paintings and radiocarbon dating, showed that there was an earlier occupation linked to the Late Archaic period.

KEYWORDS: Jequetepeque, Middle valley, Late Archaic, Platforms, Art Rock , Radiocarbon Dating

1.- Doctor de arte y ciencia de la Universidad de Tokio, Director del Proyecto Tembladera, actualmente labora en el Museo Universitario de la Universidad de Tokio.

2.- Licenciado en arqueología de la Universidad Nacional Mayor de San Marcos, Codirector del Proyecto de Tembladera, actualmente labora en el Ministerio de Cultura. 
"La Pampa de Mosquito" se extiende aproximadamente $2 \mathrm{~km}$ a lo largo del río Jequetepeque en la margen sur, esta zona pertenece a la comunidad de Trinidad y políticamente está ubicado en el distrito de Yonán-Tembladera, provincia de Contumazá, departamento de Cajamarca (Figura 1). En este artículo presentaremos brevemente los resultados de nuestra excavación en un montículo denominado "Plataforma Z1" que está situado en el extremo este de la Pampa de Mosquito que corresponde al Periodo Arcaico Tardío.

\section{ANTECEDENTES DE INVESTIGACIÓN}

En los años de 2003, 2004 y 2005 el Dr. Eisei Tsurumi, coautor del presente artículo, dirigió excavaciones arqueológicas en sitios del periodo Formativo ubicados entre los poblados modernos de Tembladera y Las Huacas, principalmente en la Pampa de las Hamacas situada en la margen norte del valle medio de Jequetepeque (Cholán et al. 2006, Tsurumi et al. 2003, Tsurumi et al. 2005, Tsurumi en prensa). Con el apoyo de los anteriores estudios realizados en la zona (Carcelén 1984, Keatinge 1980, Paredes 1984, Ravines 1981, 1982, 1985a, 1985b, Ravines y Matos 1983, Tam y Aguirre 1984, Tellenbach 1986, Ulbert 1994), los resultados de las excavaciones y prospecciones dilucidaron una larga secuencia de ocupación temprana del Periodo Formativo.

Se concluyó la existencia de dos fases durante este periodo: Fase Hamacas (Formativo Temprano, 1500-1250 a.C. (calib.) y Fase Tembladera (Formativo Medio, 1250-800 a.C. (calib.)). En este espacio de tiempo se erigieron varios conjuntos arquitectónicos formados por plataformas y grandes plazas, a los cuales Tsurumi denominó "Complejo Hamacas". Estos conjuntos del Complejo Hamacas se agruparon en nueve unidades de centros ceremoniales y la secuencia de apertura y clausura de cada centro ceremonial representa el proceso de cambio social de esta región (Tsurumi 2010, 2014). Merece especial atención señalar que los habitantes de la fase Hamacas, la primera ocupación en la Pampa de las Hamacas, elaboraron la cerámica más temprana del valle (Ulbert 1994).

Sin embargo, existe un petroglifo en la Pampa de Mosquito, localizado en la margen opuesta a la Pampa de las Hamacas, que sugiere la posibilidad de hallar alguna evidencia de asentamientos aún más tempranos debido a que este petroglifo presenta características estilísticas asociadas al Periodo Arcaico Tardío.

En el extremo oeste de la Pampa de Mosquito se presenta una densa concentración de petroglifos denominada "Quebrada del Felino" (Pimentel 1986) y entre ellos se encuentra una imagen de grandes dimensiones que denominamos "Felino" (Figura 2), que presenta características similares al arte del Arcaico Tardío (Bischof 1994) registrado en varios sitios arqueológicos mayormente del departamento de Ancash (Falcón y Suarez 2009).

En el año 2009 los autores de este artículo excavaron la "Plataforma B1" y "Plataforma C1" de Pampa de Mosquito, que son dos montículos pequeños ubicados próximos al "Felino" (Figura 3) y no presentan cerámica en superficie. La Plataforma B1 está construida modificando una colina natural, mientras tanto la Plataforma C1 está situada en la llanura y presenta un montículo pequeño casi circular de $20 \mathrm{~m}$ de diámetro y $2 \mathrm{~m}$ de altura, aunque originalmente presentaba configuración cuadrangular.

Las excavaciones iniciadas en estas dos plataformas concluyeron que tampoco existe cerámica en los rellenos lo cual sugiere que corresponderían al Arcaico Tardío, igual que el estilo plasmado en el petroglifo "Felino" (Tsurumi y Morales 2010); el detalle de estas excavaciones será presentado en una próxima publicación. De igual forma, como parte de estas investigaciones, se logró definir que durante la Fase Tembladera un petroglifo fue grabado próximo al "Felino" (Tsurumi y Morales 2013) y 


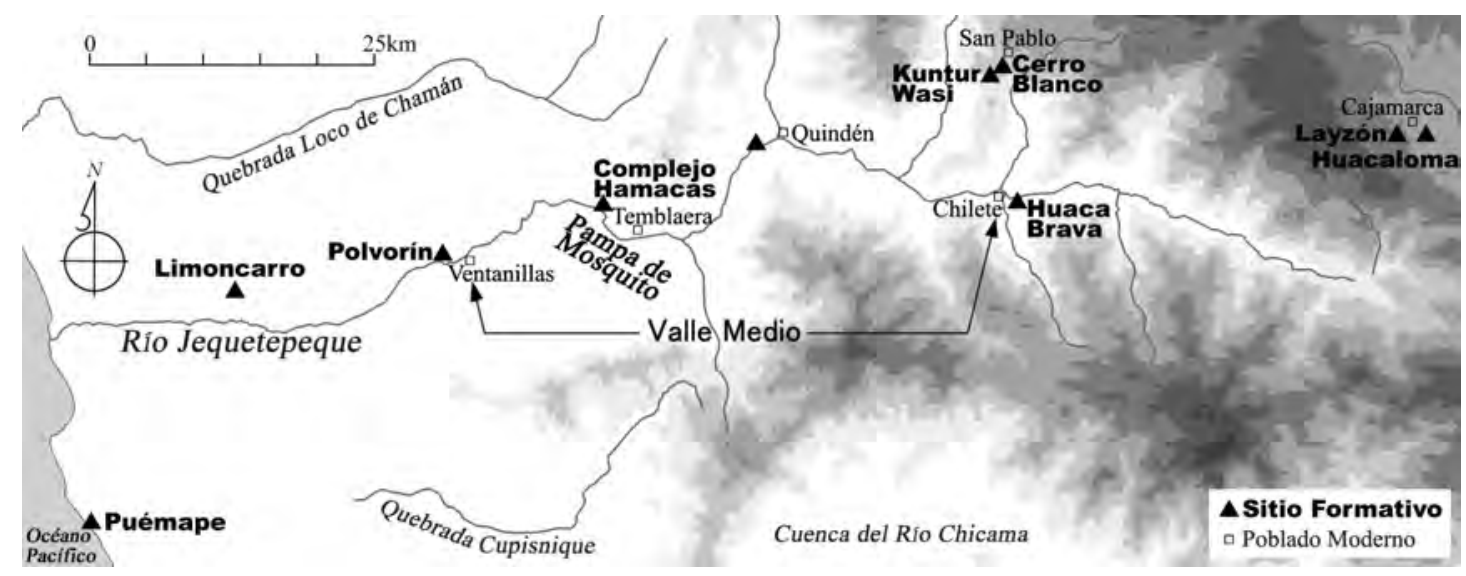

Figura 1: Valle medio de Jequetepeque y sitios formativos principales

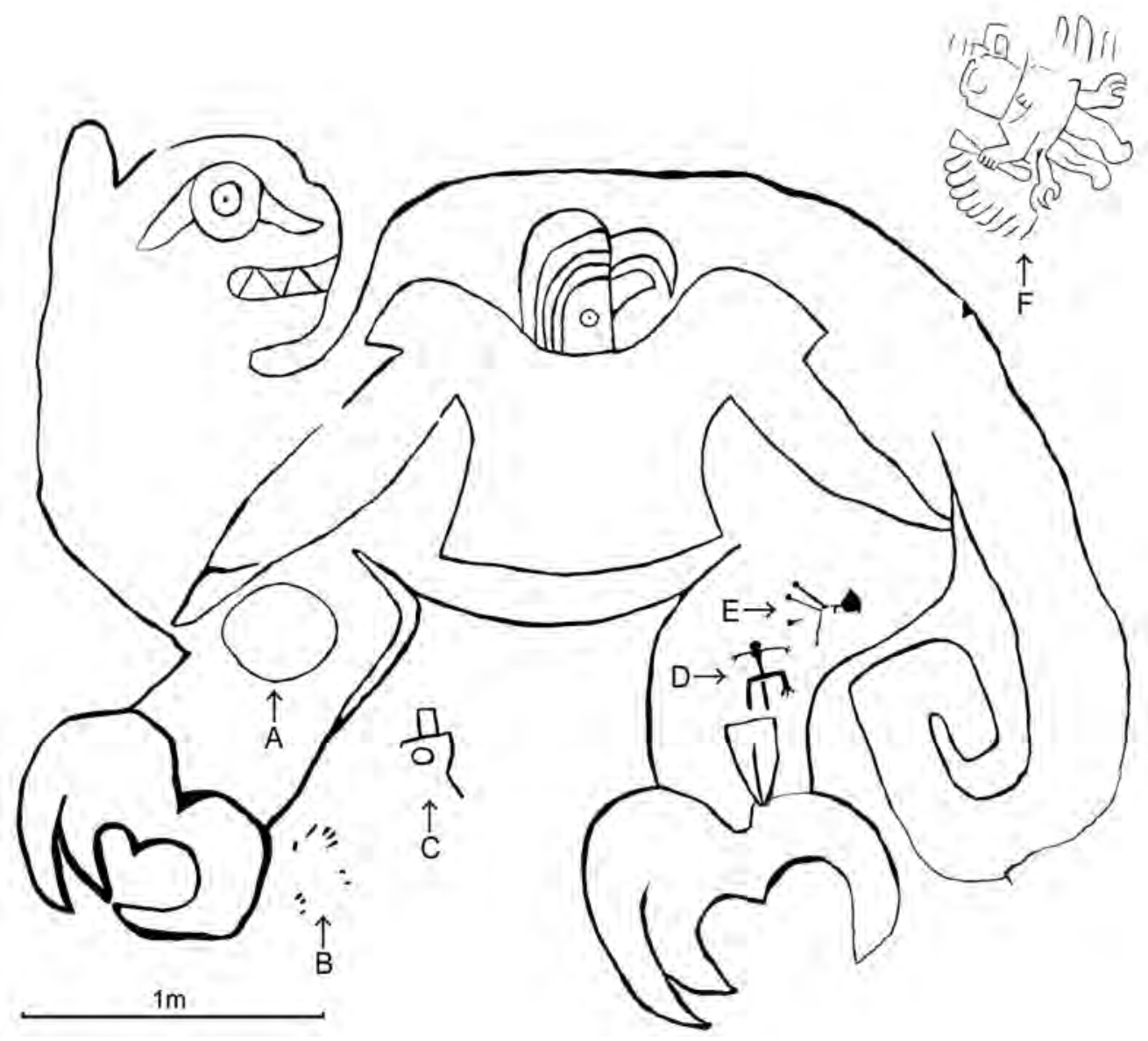

Figura 2: El petroglifo Felino y grabados agregados posteriormente en su contorno e interior (A-F): la imagen $F$ corresponde a la Fase Tembladera 


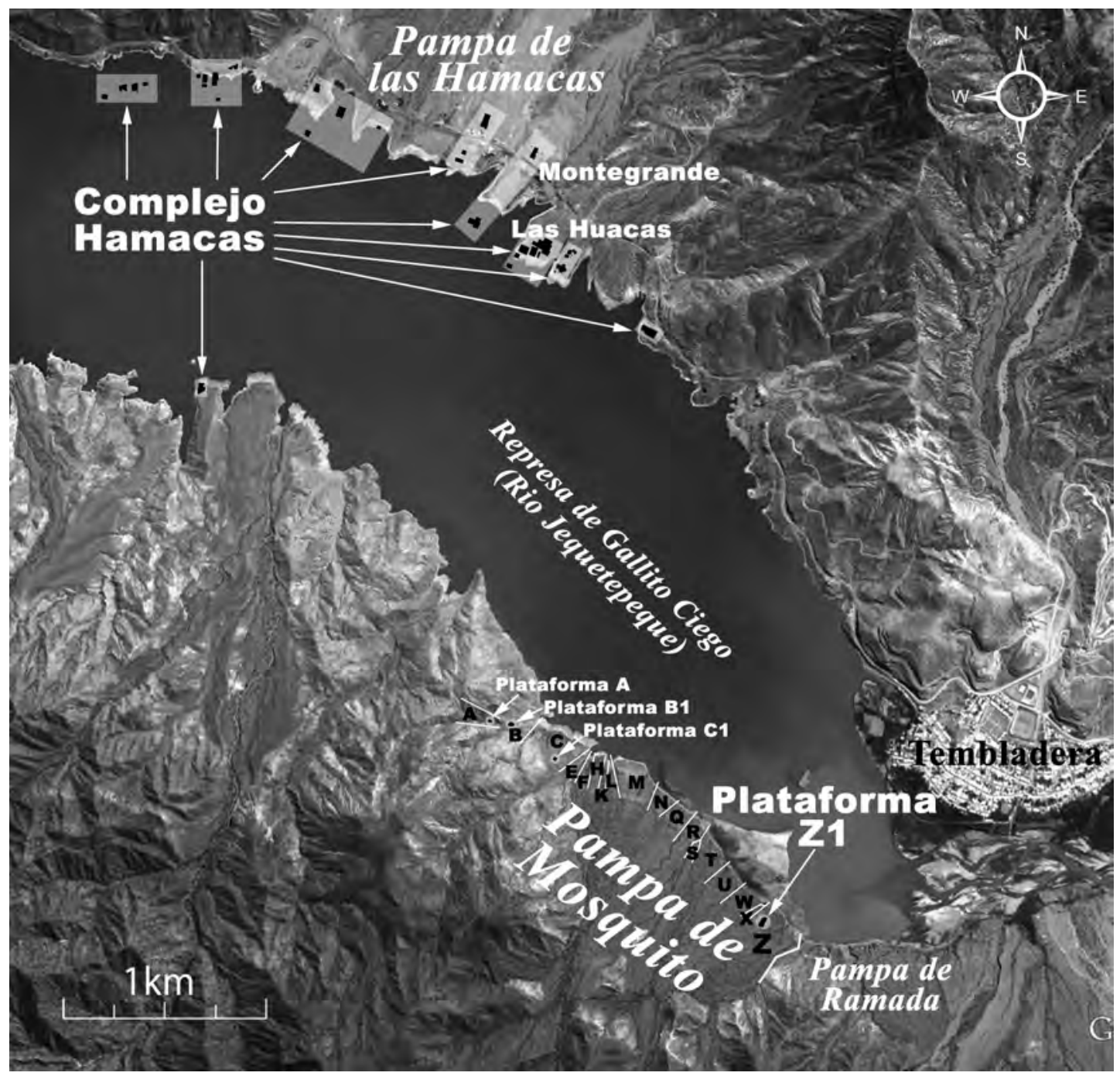

Figura 3: La Pampa de Mosquito y la Pampa de las Hamacas 
a la Plataforma A (Figura 3), que es una plataforma amorfa, edificada en frente de la roca del "Felino" (Tsurumi y Morales 2012b).

Detectar evidencias arqueológicas que preceden a la Fase Hamacas es importante para poder aclarar el proceso de formación de asentamientos con edificios monumentales no solo a nivel local, sino también en un nivel regional, debido a que no hay evidencia certera de la presencia de arquitectura monumental del Arcaico Tardío en el valle de Jequetepeque (Castillo 2009: 40). A partir de esta problemática programamos otra excavación en otro montículo sin cerámica en la Pampa de Mosquito en el año 2011.

\section{La Pampa de Mosquito y la Plataforma Z1}

La Pampa de Mosquito es un abanico aluvial con declive hacia el norte, varias quebradas hondas delimitan mesetas alargadas en dirección (aproximadamente) de N-S. Considerando la forma del terreno hemos dividido la Pampa de Mosquito en dieciocho "Áreas" (Figura 3).

El "Área A" corresponde a una quebrada donde se sitúa el petroglifo "Felino". El "Área B" es el contorno de la boca de dicha quebrada donde se concentran la mayoría de los petroglifos de la "Quebrada del Felino". El "Área C" es una meseta muy amplia con declive suave hacia el rio. La Plataforma B1 se ubica en el Área B y la Plataforma C1 en el Área C. Actualmente las mesetas del lado oeste (Área B y C), bajan suavemente hacia el rio. En la parte media de la pampa (entre las Áreas E y R) hay un declive pronunciado y la parte este de la pampa (entre las Área T y Z) presenta un acantilado. Los pobladores locales quienes conocen la zona antes de la construcción de la represa Gallito Ciego señalan que todas las mesetas originalmente presentaban un barranco, lo cual sugiere que el terreno fue alterado por causa de la represa y algunos restos arqueológicos que se localizaban cerca del rio colapsaron.

Las investigaciones ejecutadas antes de la construcción de la Represa de Gallito Ciego principalmente se realizaron en la margen norte del rio. Las publicaciones que mencionan a la Pampa de Mosquito son escasas; solamente es mencionado en los estudios sobre petroglifos de la Quebrada del Felino y se hace mención al nombre de "El Mosquito" como zona de procedencia de cerámica formativa (Alva 1986: 76, 152) y como un cementerio formativo (Alva 1986: Beilage).

En la actualidad esta pampa no está habitada y no hay acceso peatonal permanente con la margen norte, por este motivo varios sitios arqueológicos ubicados encima de la meseta se encuentran en buen estado, excepto algunos intensamente huaqueados.

En el año 2004 Tsurumi y los miembros del proyecto prospectaron la pampa e informaron de varios sitios arqueológicos (Tsurumi et al. 2005) incluyendo las plataformas B1 y C1, considerándolos como sitios de periodos tardíos por los escasos fragmentos de cerámica de estilo Gallinazo y/o Chimú que se encontraron en superficie y cerca de estos. También supusieron que la Plataforma Z1, objeto del presente artículo, pertenecería a la sociedad Chimú.

La Plataforma Z1 del Área Z se encuentra ubicada al final de la actual Represa de Gallito Ciego, al extremo este de la Pampa de Mosquito y al frente del actual pueblo de Tembladera. En el Área Z se encuentran cinco montículos (Figura 4), la Plataforma Z1 es la que presenta mayor altura y extensión, aproximadamente 10m de altura y presenta forma alargada en dirección NE-SO con una longitud aproximada de 55 metros. Esta dirección corresponde al declive natural del terreno y los ejes arquitectónicos de los cinco montículos parecen que continúan el terreno según los muros que se nota en superficie. A 80 metros al noreste de la Plataforma Z1 la pampa termina en un barranco pronunciado. Entre la Plataforma Z1 y el barranco se encuentran muros que forman recintos pequeños, varios de 
estos muros están asociados a cerámica de estilo Chimú que puede indicar la posición cronológica de estos recintos.

Como mencionamos líneas arriba, en el proyecto 2004, se concluyó que las plataformas y recintos de esta área eran contemporáneos y pertenecientes a periodos tardíos, pero luego de las excavaciones en las Plataformas B1 y C1 se sospechó de la veracidad de esta interpretación. Las superficies de estas dos plataformas están cubiertas con piedras angulosas y canteadas, las excavaciones arrojaron como resultado que gran cantidad de este material fue amontonado para formar la plataforma y expuestos después del colapso. La superficie de la Plataforma Z1 es similar a la B1 y C1, y diferente de las plataformas formativas de las fases Hamacas y Tembladera que sus rellenos están compuestos básicamente con tierra.

Considerando dichas características, hipotéticamente consideramos que la Plataforma Z1 pertenece al Arcaico Tardío. Sin embargo, la Plataforma Z1 es distinta a la Plataforma B1 en cuanto a su emplazamiento en el espacio, y presenta mayor dimensión que la Plataforma C1. Sobre todo, es importante indicar que la Plataforma Z1 forma parte de un conjunto arquitectónico muy extenso que consiste en cinco plataformas colindantes dentro del Área Z (Figura 4), las Plataformas B1 y C1 están ubicados aisladamente. Ante estas peculiaridades decidimos excavar la Plataforma Z1 para confirmar su posición cronológica y compararlo con los datos del otro extremo de la pampa obtenidos en nuestro proyecto de la temporada 2011 (Tsurumi y Morales 2012a).

\section{Excavaciones en la Plataforma Z1}

Como estrategia de excavación nos propusimos descubrir la superposición de varios muros y pisos, y obtener muestras de carbón asociadas a ellos para aclarar las fases constructivas y sus fechados. Seleccionamos la ladera empinada del lado noreste de la plataforma porque alcanzamos a observar varias superposiciones de muros dentro de pozos de huaqueo. La excavación se realizó a través de unidades de $2 \mathrm{~m}$ por $2 \mathrm{~m}$ y se realizaron en total diez unidades profundizándolas hasta las capas estériles (Figura 5, Figura 6 y Figura 7). En algunas unidades no pudimos profundizar hasta llegar a niveles de piso o capa estéril por falta de espacio entre los muros. Además, como estaba previsto, los estratos contienen gran cantidad de piedras (véase Figura 6) a punto de desmoronarse que fue un obstáculo a la hora de la excavación.

El resultado de nuestra excavación nos permite inferir la presencia de, al menos, tres fases constructivas marcadas por la renovación de muros principales de contención que sostienen el lado noreste de la plataforma. En este artículo las mencionaremos como la "Fase 1", la "Fase 2" y la "Fase 3". Esta asignación aún es provisional; teniendo en cuenta que en niveles más profundos podría haber otros edificios aún anteriores a la Fase 1. La Fase 2 se dividió en seis subfases teniendo las modificaciones de plataformas superiores en cuenta. Fechamos seis muestras de carbón extraídos de contextos arqueológicos a través de AMS. Para la calibración de fechados utilizamos el programa OxCal v.4.2.3 (Bronk Ramsey 2013) y especialmente de cuatro muestras asociados directamente al edificio dedicaremos un párrafo más adelante.

En la Fase 1 (Figura 7 y Figura 8-A) la cara noreste de la Plataforma Z1 fue sostenida por el muro ZM18 y el lado sureste por el muro ZM12. Cabe decir que hemos descubierto la esquina este de la plataforma si su forma es simplemente rectangular. No ubicamos elementos arquitectónicos encima de la plataforma. El muro ZM18 presenta una escalinata angosta y empinada denominada ZE1 con seis peldaños y los dos peldaños inferiores sobresalen de la cara del muro ZM18. Al costado noroeste de la escalera ZE1 seleccionamos una muestra de carbón sobre el piso (11MQZ-A-C9; Beta-322915) que dio como resultado un fechado de $3480 \pm 30 \mathrm{BP}$. 


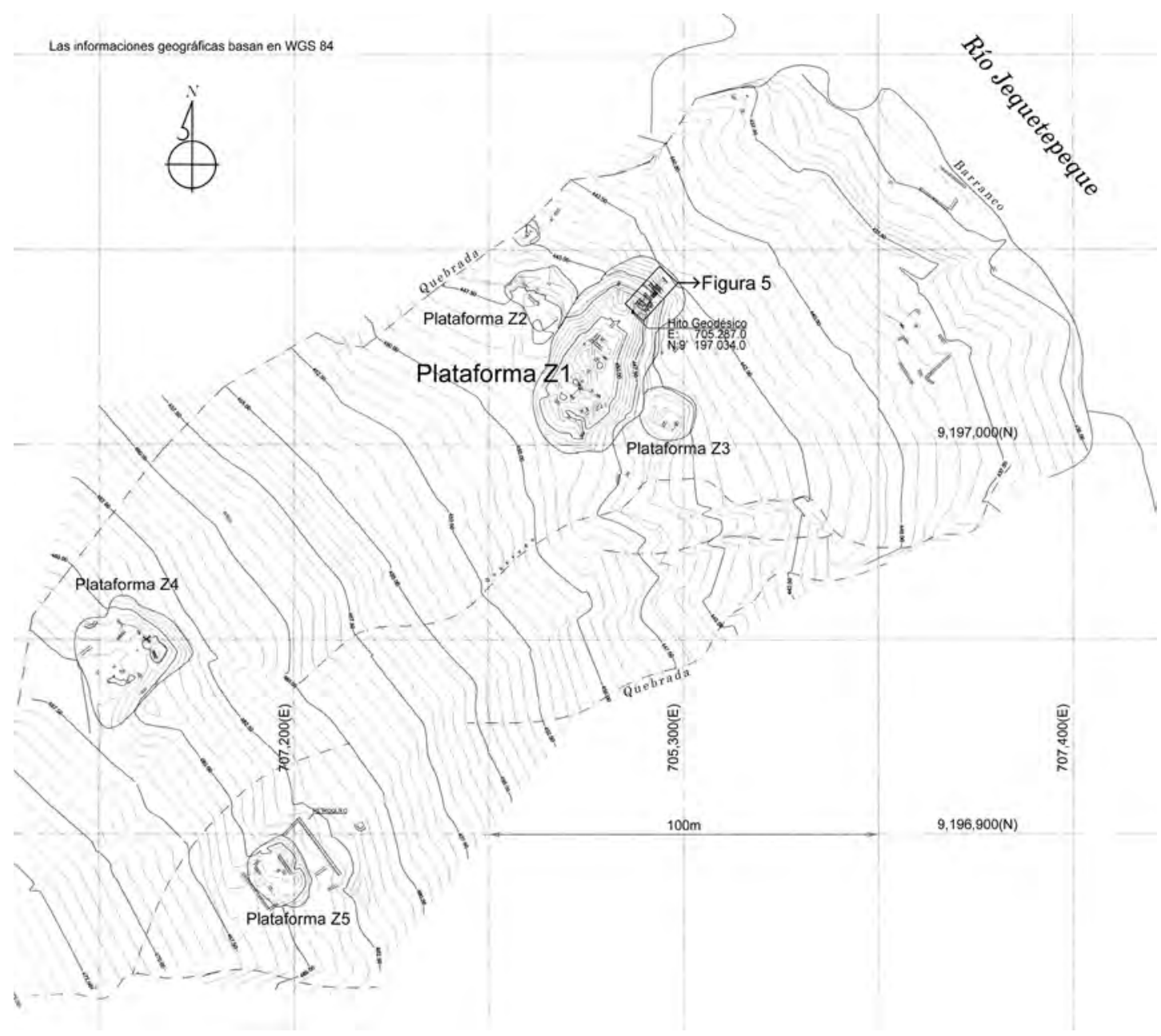

Figura 4: La Plataforma Z1 ubicado en el Área Z 


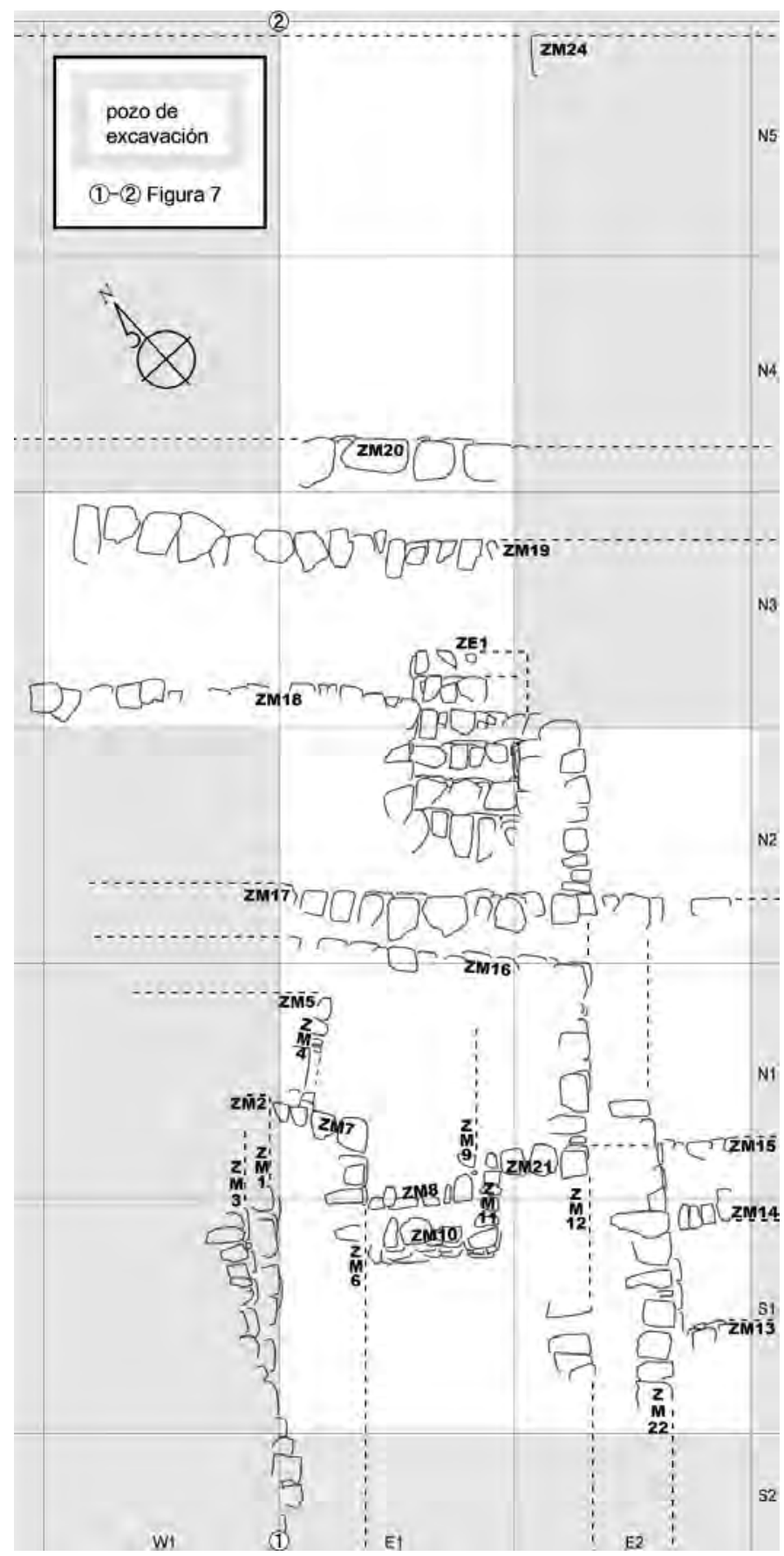

Figura 5: La zona de excavación en la Plataforma Z1 


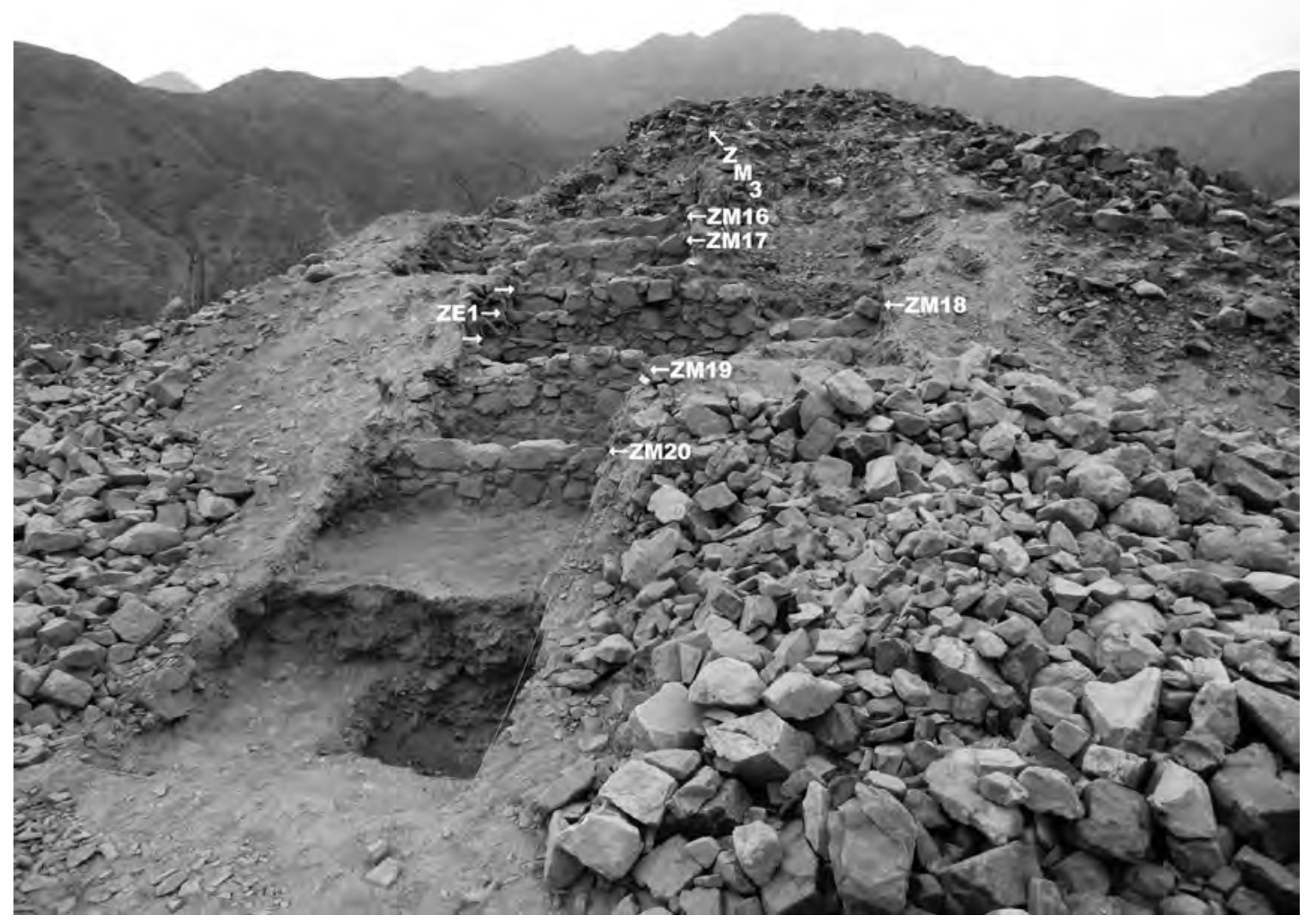

Figura 6: Vista de la excavación en la Plataforma Z1 tomada desde el noreste: las piedras amontonadas a este lado fueron extraídas de las capas excavadas 
En la segunda fase, el muro ZM19 fue construido para sostener la cara noreste de la plataforma; el muro ZM18 y la escalera ZE1, de la fase anterior, fueron cubiertos por el piso que conecta los muros ZM19 y ZM17 (Figura 7 y Figura 8-B). El nuevo muro de contención del lado sureste que forma la esquina junto con ZM19 debe estar más al sureste de nuestra zona de excavación. El extremo noreste del muro ZM12 posiblemente formó esquina junto con el muro ZM17 mientras la parte suroeste de ZM12 fue cubierta por un muro nuevo denominado ZM15 que mira hacia noreste. Los muros ZM17, ZM12 y ZM15 sostienen un piso de nivel -353 y encima de este piso hemos hallado la esquina este de una plataforma superior formada por los muros ZM2 y ZM1. Esta es "la Fase 2a", la primera subfase de la Fase 2. De acuerdo a la superposición de los muros encima del piso-353 necesitamos presumir la existencia de cuatro subfases más como lo siguiente; en la Fase 2b los muros ZM5 y ZM4 fueron construidos para ampliar la plataforma superior (Figura 8-C), ZM7 y ZM6 en la Fase 2c (Figura 8-D), ZM9 y ZM8 en la Fase 2d (Figura 8-E) y ZM10 y ZM11 en la Fase 2e (Figura 8-F). En la Fase 2 f(Figura 8-G) la cabecera del muro ZM12 fue elevada y formó esquina con un nuevo muro denominado ZM16, y en el mismo momento posiblemente el muro ZM17 fue prolongado hacia el sureste tapando el muro ZM15. Tenemos dos fechados radiocarbónicos correspondientes a la Fase 2. Una muestra de carbón adherido al enlucido de ZM15 (11MQZ-A-C7; Beta-321447) que dio como resultado un fechado de $3470 \pm 30$ BP y suponemos que corresponde al momento de cubrirse el muro ZM15 o sea la Fase 2e. Otra muestra de carbón asociado al piso entre ZM19 y ZM17 (11MQZ-A-C5; Beta-321446) resultó un fechado de $3440 \pm 30 \mathrm{BP}$ a cual hipotéticamente consideramos como fecha de la Fase 2f, es decir, inmediatamente antes de cubrirse por el piso de la Fase 3.

Las evidencias de la Fase 3, la última fase, se encuentran dispersos debido al colapso de la plataforma (Figura 7 y Figura 8-H). Debemos suponer que el muro ZM20 apareció para sostener el lado noreste de la plataforma cubriendo el muro ZM19, recalcamos que su estado de conservación es malo. Los muros de las plataformas superiores construidos durante varias subfases de la Fase 2 fueron revestidos por un piso de nivel -215 que originó una nueva plataforma superior sostenida por el muro ZM3. Aún no podemos afirmar con seguridad que el piso -215 se extiende de manera horizontal hasta la cabecera del muro ZM20 como mostramos hipotéticamente en la Figura 7. En el lado sureste el muro ZM12 fue cubierto por el muro ZM22 que está asociado a los muros ZM14 y ZM13, son dos muros paralelos que forman un espacio angosto y apisonado. Una capa gruesa de ceniza fue colocada encima de la capa estéril antes de construir el muro ZM20 y se recogió una muestra de carbón de esta capa (11MQZ-A-X7; Beta-321450) resultando un fechado de 3420ะ30BP. Encima de dicho piso se acumuló otra capa de ceniza que corresponde al momento posterior a la Fase 3, realizamos el análisis radiocarbónico de la muestra de carbón (11MQZ-A-X8; Beta-321451) arrojando un fechado de 3460ะ30BP (1880-1620 a.C., $\square 2$ calib.).

Cerca del extremo noreste de nuestra zona de excavación se encuentra una piedra que presenta cara hacia el noreste. Hipotéticamente la consideramos como parte de muro denominado ZM24, las capas de su contorno son delgados y no nos permite inferir la correspondencia con los muros de contención de cada fase; por el momento lo situamos en la Fase 3 (Figura 7 y Figura 8-H).

\section{Cronología de la Plataforma Z1}

Es necesario mencionar que hay evidencias arqueológicas encontradas en el área de investigación perteneciente a periodos posteriores. La observación superficial realizada en el año 2004 y antes de las excavaciones del 2011 detectó escasos fragmentos de cerámica Chimú, y nuestra excavación de la primera capa dio como resultado la aparición de pocos fragmentos formativos. Todos los fragmentos formativos son de cuerpo de botella fina sin decoración (Figura 9). Dieciséis fragmentos proceden de 


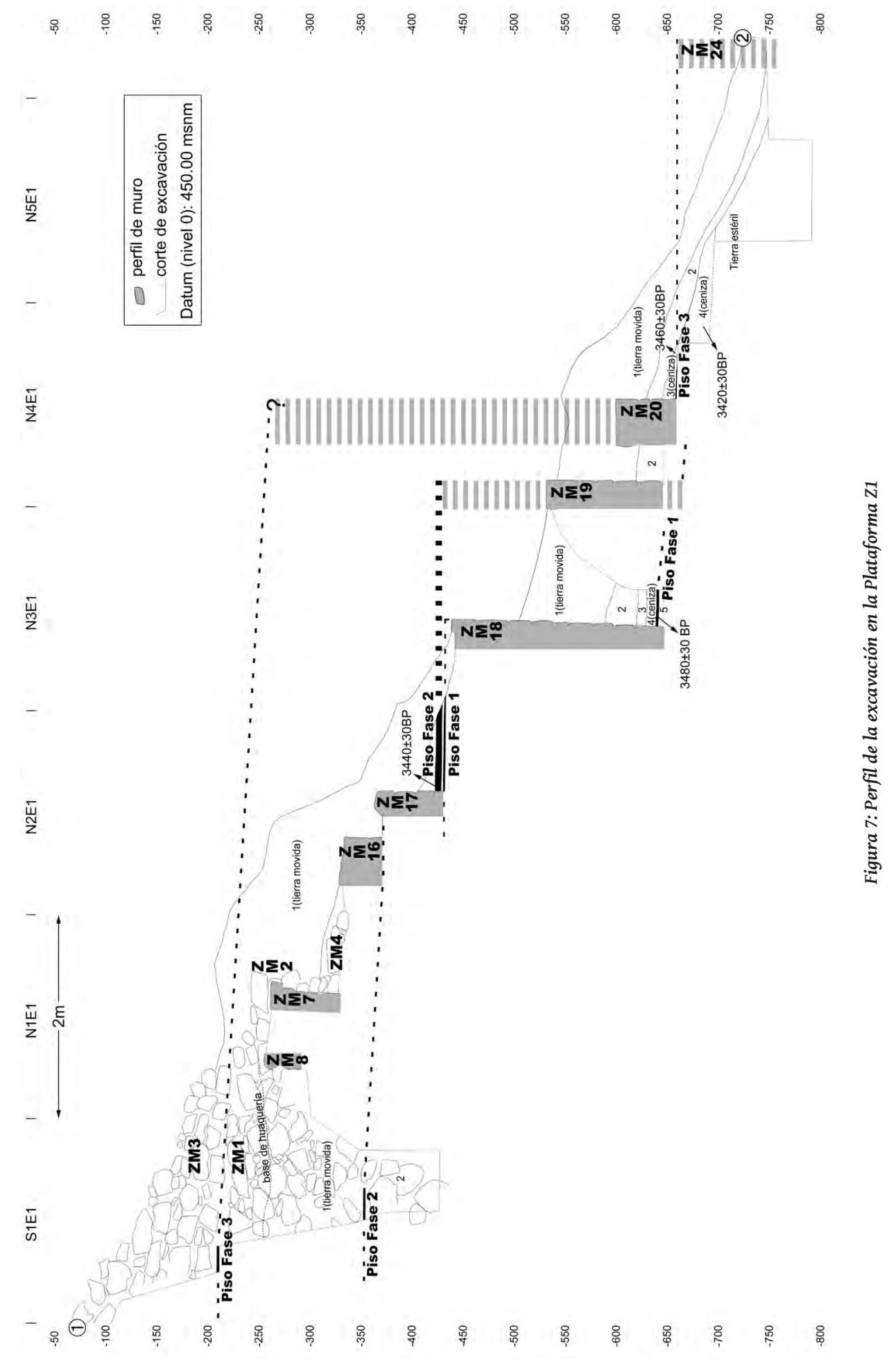


Figura 8A - 8H: Fases (y subfases) constructivas de la Plataforma Z1

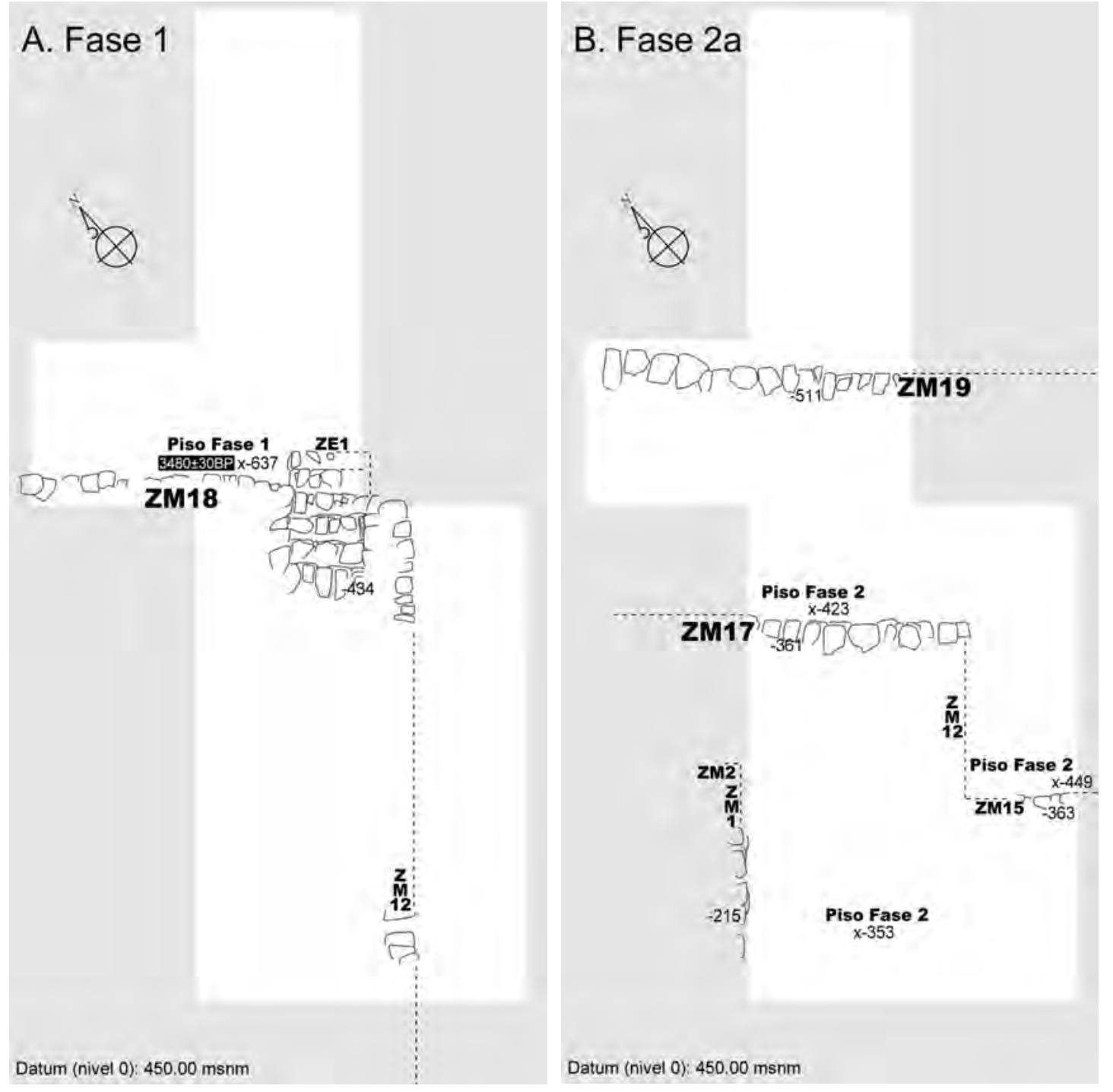




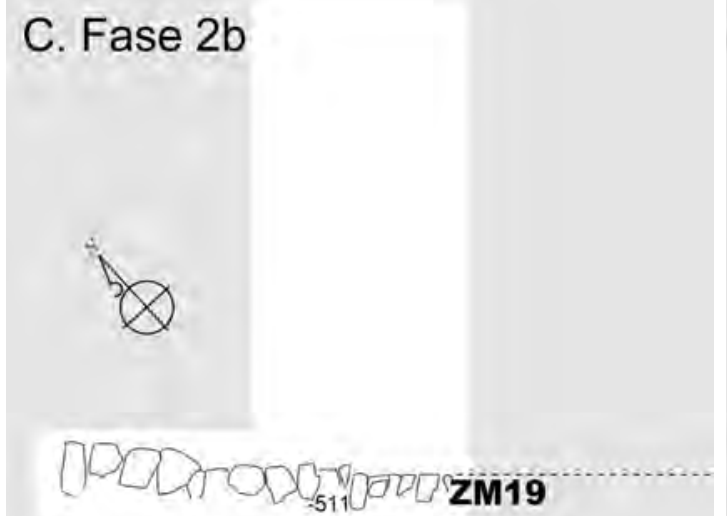

D. Fase 2c
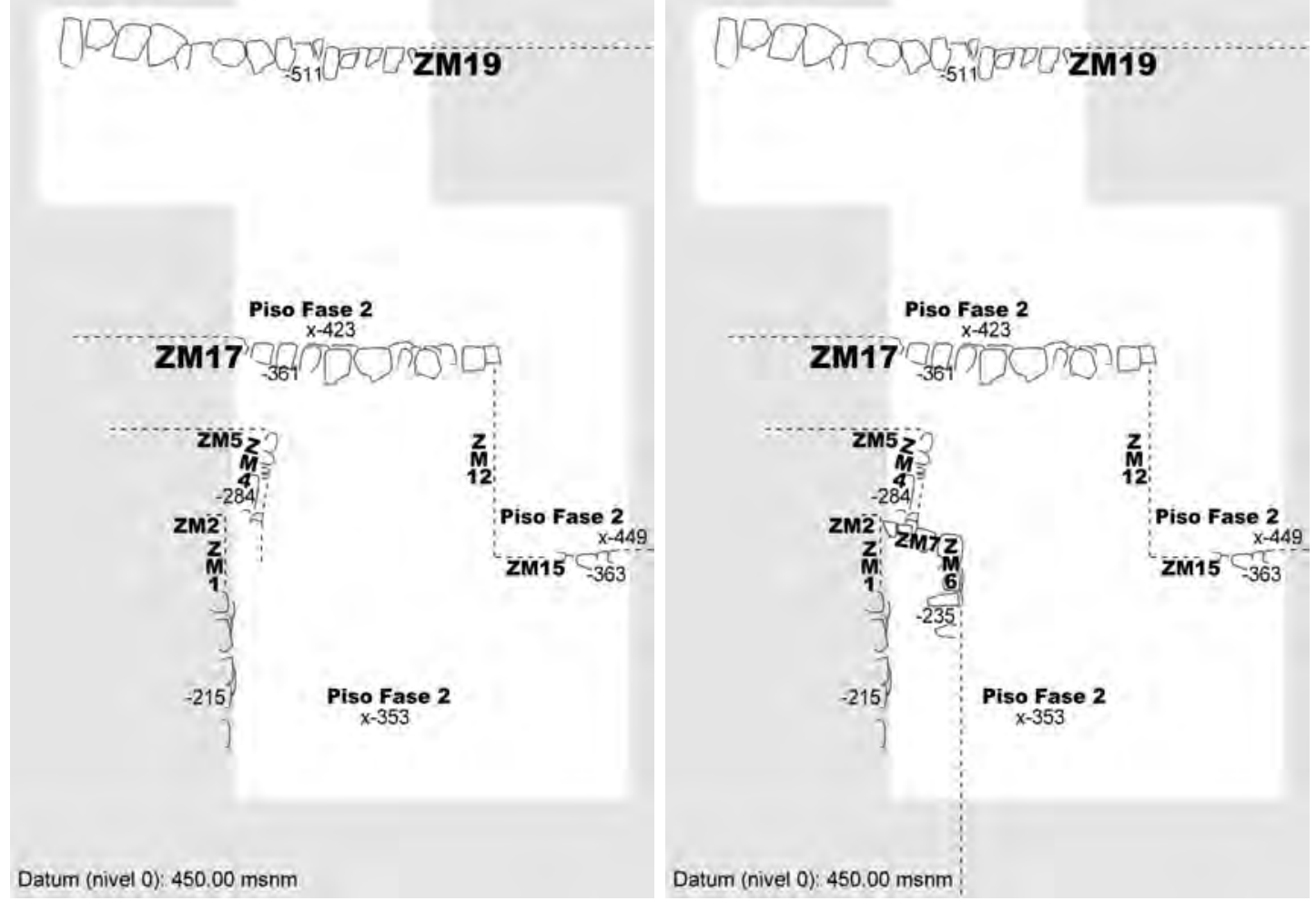

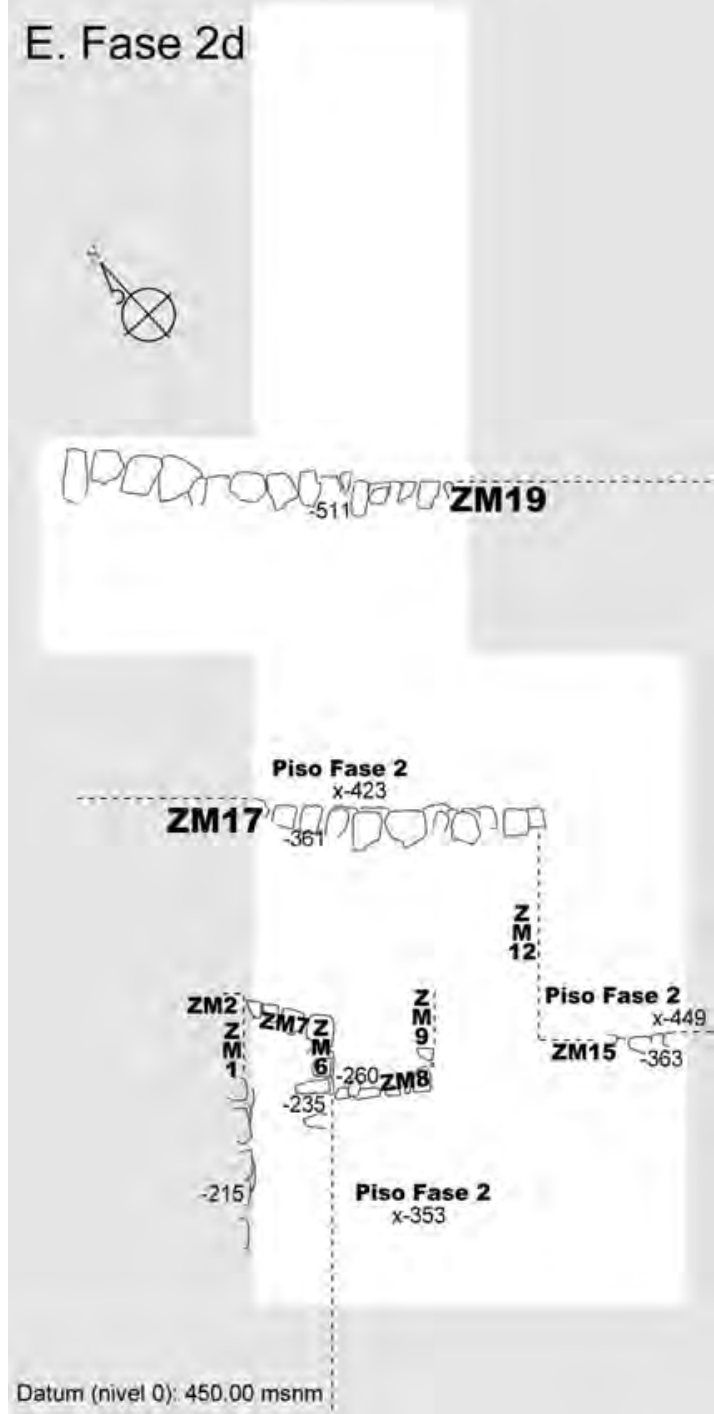

F. Fase $2 e$
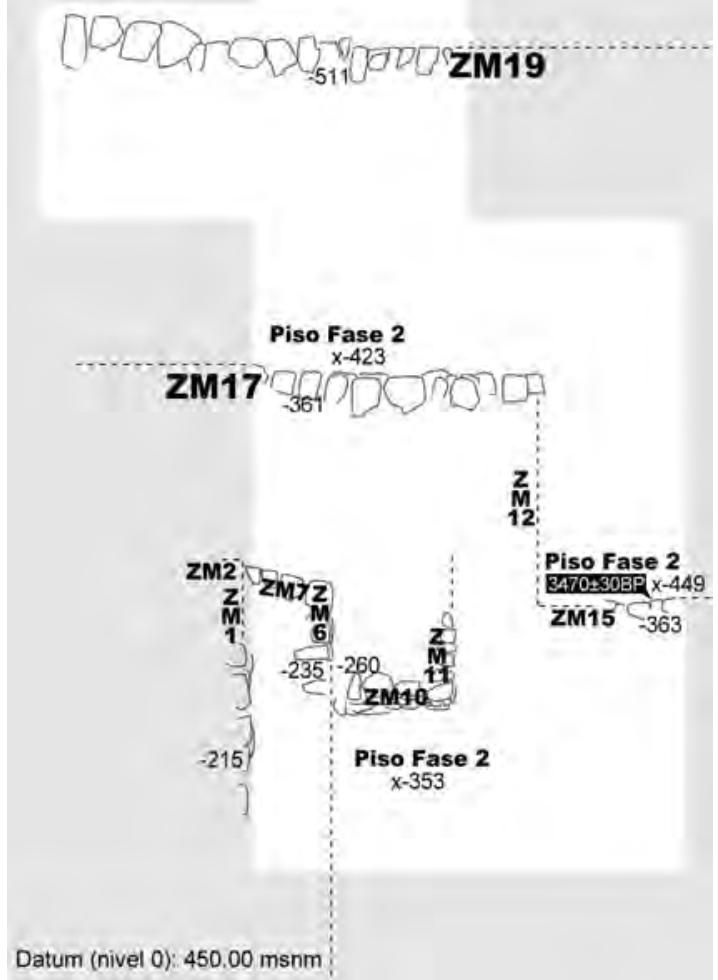

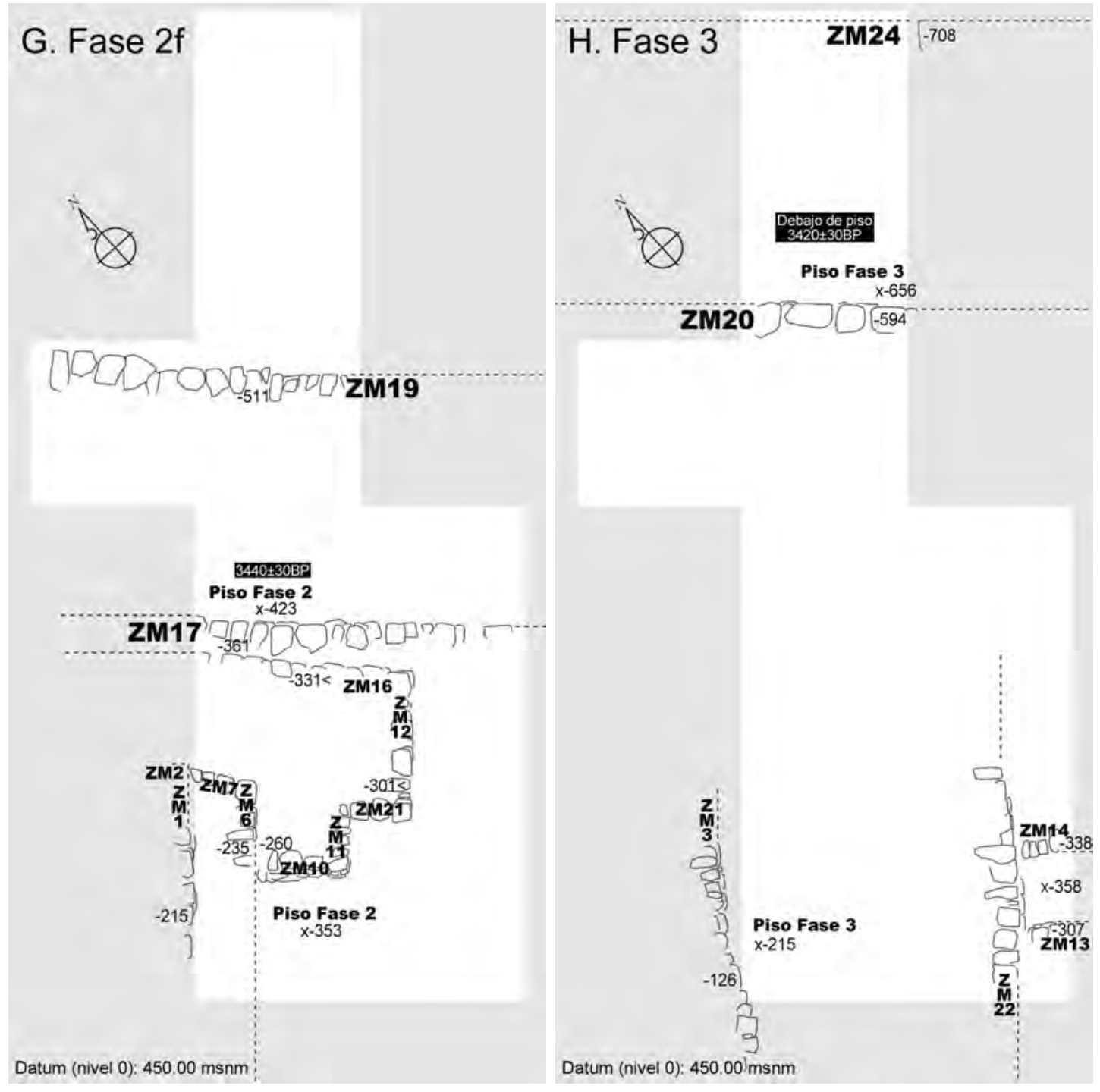
dos o tres botellas de cuerpo globular de la Fase Tembladera (Formativo Medio) y una botella de cuerpo cilíndrico con base plana que puede corresponder a la Fase Lechuzas (Formativo Tardío, 800-550 cal a.C.). Estos materiales nos recuerdan el caso de Montegrande, donde se encontraron fragmentos de cerámica muy fina de estilos posteriores en la superficie del sitio de la Fase Hamacas (Ulbert 1994: 148); Tsurumi infiere que este hallazgo es parte de restos de ofrendas de una ceremonia realizada durante la Fase Tembladera en los monumentos clausurados de periodos anteriores (Tsurumi 2014: 208-209).

Una muestra de carbón fue tomada de la superficie del piso que corresponde a la cabecera del muro ZM18 (Figura 7), esperábamos que el resultado nos diera una fecha relacionada a la Fase 1

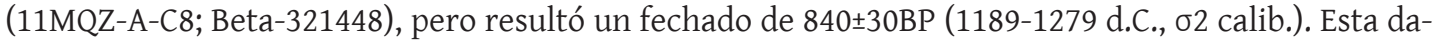
tación fue un fracaso por nuestro descuido en muestrear carbón asociado al piso parcialmente roto y cubierto de la primera capa, es decir, este contexto es resto de alguna actividad destructiva realizada en periodos tardíos, posiblemente durante la ocupación de la sociedad Chimú.

La ausencia de cerámica, excepto las halladas en la primera capa, sugiere que la Plataforma Z1 corresponde al Arcaico Tardío y la datación radiocarbónica prueba esto. Estimamos que los carbones asociados a la cara de muros y sobre los pisos corresponden a momentos finales, suponiendo acciones de limpieza durante el uso del edificio, y la ceniza muestreada debajo del piso fue depositada antes de su construcción. Al realizar los fechados calibrados ( $\sigma 2$ calib.) se aplicó el análisis bayesiano (Bronk Ramsey 2009) para reducir el margen error de cuatro muestras que corresponden a diferentes fases (y subfases) constructivas, que son las siguientes: (Figura 10).

(Final de) Fase 1: 11MQZ-A-C9 (Beta-322915): 1875-1675 a.C.

(Final de) Fase 2e: 11MQZ-A-C7 (Beta-321447): 1780-1660 a.C.

(Final de) Fase 2f: 11MQZ-A-C5 (Beta-321446): 1750-1640 a.C.

(Inicio de) Fase 3: 11MQZ-A-X7 (Beta-321450): 1740-1610 a.C.

Solamente con cuatro fases no se puede reducir satisfactoriamente el margen para determinar fechas con absoluta precisión, por lo tanto en futuros trabajos excavaremos la parte nuclear de la plataforma y de esta manera confirmar fases constructivas anteriores a la Fase 1, y obtener muestras para nuevos fechados. Por el momento estimamos que la secuencia cronológica desde la Fase 1 hasta la Fase 3 se encuentra en un lapso de tiempo que va entre 1800 y 1600 a.C.

\section{Comentarios Finales}

Como conclusión de este breve articulo queremos afirmar que en la Pampa de Mosquito del valle medio de Jequetepeque se halló un monumento arqueológico del Arcaico Tardío, probablemente un centro ceremonial debido a su dimensión y disposición arquitectónica. A esta época de ocupación del Arcaico Tardío en un nivel local la denominaremos "Fase Mosquito" (termina en 1500 cal a.C.).

Las evidencias de la Fase Mosquito pueden ofrecernos oportunidades de profundizar en el debate sobre la formación de las sociedades complejas en esta parte de los andes. Al respecto, primero, necesitamos considerar cuando y como se formaron estos monumentos arquitectónicos tempranos en el valle de Jequetepeque, porque la aparición de las primeras ocupaciones con monumento arquitectónico es un tema esencial de la arqueología Centroandino. 
Segundo, nos interesa averiguar las razones del abandono de esta ocupación. En la pampa de Mosquito se encuentran evidencias de las actividades durante la Fase Tembladera, tanto en el contorno del petroglifo "Felino" (Tsurumi y Morales 2012b) como las posibles ofrendas en la Plataforma Z1. Sin embargo, la evidencia de la Fase Hamacas, la fase inmediatamente después de la Fase Mosquito, es notablemente escasa; hasta ahora tres fragmentos de olla recuperados en el Área T son los únicos elementos culturales de la Fase Hamacas. El abandono de las ocupaciones en la Pampa de Mosquito y la apertura de asentamientos en la otra margen, Pampa de las Hamacas, es un cambio evidente de patrón de asentamiento. Aprovechando los datos sobre las fases siguientes acumulados por nuestros proyectos pasados podremos enfrentarnos a este problema.

Para discutir estos problemas generales necesitamos investigar minuciosamente en la Pampa de Mosquito a través de análisis arquitectónico y radiocarbónico. Primero debemos aclarar las relaciones entre la Plataforma Z1 y los otros montículos del Área Z; como lo sucedido en el Complejo Hamacas las cinco plataformas del Área $Z$ pueden formar una unidad de centro ceremonial. Segundo, tenemos que comprender la totalidad de la ocupación de la Fase Mosquito. No solamente en Áreas A, B, C y Z sino en otras mesetas del extremo norte de la pampa donde se encuentran montículos sin cerámica formativa y cubiertos de piedras. Además, en la parte alta de la pampa ubicamos varios montículos, algunos son más monumentales que la Plataforma Z1.

A propósito al este del Área Z cruzando una quebrada honda comienza otra pampa que se llama Pampa de Ramada y allí también se encuentran montículos similares. La extraordinaria extensión de los sitios arqueológicos en la zona es un obstáculo de investigación, pero eso ofrece varios puntos de vista en el estudio de patrón de asentamiento y el paisaje. No solamente nos enfocamos en monumentos arquitectónicos, sino prestamos atención a otros elementos culturales y ambientales como zonas residenciales, campo de cultivo, arte rupestre, recursos naturales y características geográficas del terreno. A partir del año 2014 iniciaremos una nueva temporada del proyecto.

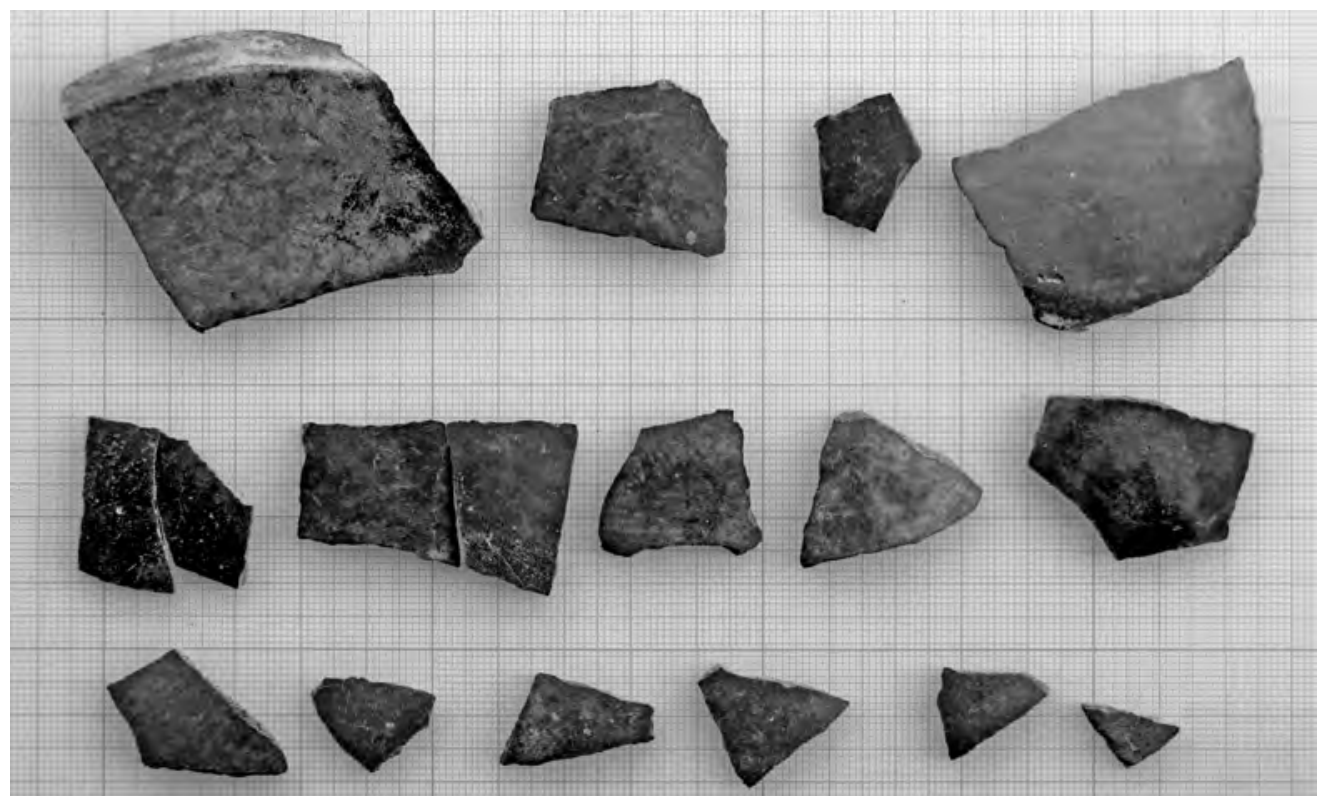

Figura 9: Fragmentos de cerámica formativa recuperados de la Capa 1: lo fragmentos ubicados arriba a la izquierda corresponde a la Fase Lechuzas y los demás a la Fase Tembladera 


\section{Agradecimientos}

El estudio presentado aquí se realizó con el apoyo de The Grants-in-Aid for Scientific Research del Gobierno del Japón. Agradecemos a Luís Alfredo Fernández Wanda (Universidad Nacional Federico Villarreal), nuestro asistente de campo, al Dr. Takayuki Omori (Laboratorio de Datación Radiocarbónica, Museo Universitario de la Universidad de Tokio) quien nos dio consejos sobre la interpretación de fechados radiocarbónicos aplicando la estadística bayesiana. Queremos expresar nuestro reconocimiento al Ministerio de Cultura y a la Dirección Desconcentrada de Cultura Cajamarca, por las facilidades proporcionadas a nuestro trabajo. Por último expresamos muestra profunda gratitud a La Misión Arqueológica Japonesa, por su apoyo total al presente estudio.

\section{BiBLIOGRAFÍA}

ALVA, Walter

1986 Frühe keramik aus dem Jequetepeque-Tal, Nordperu/Cerámica temprana en el valle de Jequetepeque, norte del Perú. C.H. Beck.

BISCHOF, Henning

1994 Toward the Definition of Pre- and Early Chavin Art Styles in Peru. Andean Past 4: 169-228.

BRONK RAMSEY, Christopher

2009 Bayesian analysis of radiocarbon dates. Radiocarbon 51(1): 337-360.

2013 OxCal Program, version 4.2 (http://c14.arch.ox.ac.uk/oxcal.html), Radiocarbon Accelerator Unit, University of Oxford.

\section{CARCELÉN SILVA, José}

1984 Los trabajos realizados en la Huaca Campos de Montegrande. AVA-Beiträge 6: 520-540.

CASTILLO, Luis J.

2009 La arqueología del valle de Jequetepeque y la colección Rodríguez Razzetto. En Castillo, Luis J. y Cecilia Pardo (eds.), De Cupisnique a los Incas: el arte del valle de Jequetepeque, pp. 34-61. Asociación Museo de Arte de Lima.

CHOLÁN CABANILLAS, Raúl, Eisei TSURUMI y Yasutake KATO

2006 Proyecto arqueológico: Las Huacas, valle medio de Jequetepeque, provincia de Contumazá, Depto. de Cajamarca en el 2005. Informe presentado al Instituto Nacional de Cultura.

FALCÓN HUAYTA, Victor y Mónica SUÁREZ UBILLÚS

2009 El felino en la emergencia de la civilización en los Andes Centrales. Sepúlveda R., Marcela, Luís Briones M., Juan Chacama R. (eds.), Crónicas sobre la piedra: arte rupestre de las Américas, pp. 331-348. Universidad de Tarapacá.

HOGG, Alan G., QUAN HUA, Paul G BLACKWELL, Mu NIU, Caitlin E BUCK, Thomas P GUILDERSON, Timothy J HEATON, Jonathan G PALMER, Paula J REIMER, Ron W. REIMER, Christian S. M. TURNEY and Susan R. H. ZIMMERMAN

2013 SHCal13 Southern Hemisphere Calibration, 0-50,000 Years cal BP. Radiocarbon 55(4): 18891903. 
KEATINGE, Richard W.

1980 Archaeology and Development: the Tembladera Site of the Peruvian North Coast. Journal of Field Archaeology 7: 467-475.

PAREDES ABAD, María I.

1984 El complejo sur de la Meseta 2 de Montegrande. AVA-Beiträge 6: 505-512.

PIMENTEL SPISSU, Víctor

1986 Felszeichnungen im mittleren und unteren Jequetepeque-Tal, Nord-Peru: Petroglifos en el Valle Medio y Bajo de Jequetepeque, Norte del Perú. C.H. Beck.

RAVINES, Rogger

1981 Mapa arqueológico del valle del Jequetepeque. Proyecto de Rescate Arqueológico Jequetepeque.

1982 Arqueología del valle medio del Jequetepeque. Proyecto de Rescate Arqueológico Jequetepeque/ Instituto Nacional de Cultura.

1985a Arquitectura monumental temprana del valle del Jequetepeque. Historia de Cajamarca vol. 1, Arqueología, pp. 131-146. Instituto Nacional de Cultura/ Corporación de Desarrollo de Cajamarca.

1985b Early Monumental Architecture of the Jequetepeque Valley, Peru. En C. B. Donnan (ed.), Early Ceremonial Architecture of the Andes, pp. 209-226, Dumbarton Oaks.

RAVINES, Rogger y Alejandro MATOS AVALOS

1983 Inventario de monumentos arqueológicos del Perú: zona norte. Instituto Nacional de Cultura, Lima.

TAM CHANG, Manuel e Iris AGUIRRE DE TAM

1984 El complejo Sur-Este de la Meseta 2 de Montegrande, AVA-Beiträge 6:513-519.

TELLENBACH, Michael

1986 Die Ausgrabungen in der formativzeitlichen siedlung Montegrande, Jequetepeque-Tal, Nord-Peru / Las excavaciones en el asentamiento formativo de Montegrande, valle de Jequetepeque, en el norte del Perú. C.H. Beck.

TSURUMI, Eisei

2010 La secuencia cronológica de los centros ceremoniales de la Pampa de Las Hamacas y Tembladera, valle medio de Jequetepeque. Boletín de Arqueología PUCP 12: 141-169.

2014 Un estudio de agrupaciones espaciales de centros ceremoniales formativos: el caso del Complejo Hamacas del valle medio de Jequetepeque. Senri Ethnological Studies 89: 201-223.

En prensa El período formativo en el valle medio de Jequetepeque, norte del Perú. Nayra Kunan Pacha. Revista de Arqueología Social 1. 
TSURUMI, Eisei, Regina Abraham FERNÁNDEZ y Yasutake KATO

2003 Proyecto arqueológico: Las Huacas, valle medio de Jequetepeque, provincia de Contumazá, Depto. de Cajamarca. Informe presentado al Instituto Nacional de Cultura.

TSURUMI, Eisei, Evelyn A. MORA CORONADO y Yasutake KATO

2005 Proyecto arqueológico: Las Huacas, valle medio de Jequetepeque, provincia de Contumazá, Depto. de Cajamarca en el 2004. Informe presentado al Instituto Nacional de Cultura.

TSURUMI, Eisei y Carlos A. MORALES CASTRO

2010 Proyecto arqueológico Tembladera: valle medio de Jequetepeque, provincia de Contumazá, Depto. de Cajamarca en el 2009. Informe presentado al Ministerio de Cultura.

2012a Proyecto arqueológico Tembladera: valle medio de Jequetepeque, provincia de Contumazá, Depto. de Cajamarca en el 2011. Informe presentado al Ministerio de Cultura.

2012b Plataforma con petroglifo del Periodo Formativo en la Pampa de Mosquito, valle medio de Jequetepeque. Arqueológicas Nº 29: 19-35. Revista del Museo Nacional de Arqueología, Antropología e Historia del Perú (MNAAHP).

2013 Un gato con muchas vidas: un petroglifo Arcaico Tardío en el valle medio de Jequetepeque. Mundo de Antes $\mathrm{N}^{\circ}$ 8. Revista del Instituto de Arqueología y Museo de la Universidad Nacional de Tucuman.

ULBERT, Cornelius

1994 Die keramik der formativzeitlichen siedlung Montegrande, Jequetepequetal, Nord-Peru. Philipp von Zabern. 